Southern Illinois University Carbondale

OpenSIUC

Publications

Department of Physics

$5-2003$

\title{
Magnetic Behavior of Iron-Oxoclusters Prepared in an Organosilica Sol-Gel Matrix
}

\section{S. Dubenko}

Southern Illinois University Carbondale

M. S. Rao

Southern Illinois University Carbondale

S. Roy

Southern Illinois University Carbondale

B. C. Dave

Southern Illinois University Carbondale

N.Ali

Southern Illinois University Carbondale

Follow this and additional works at: http://opensiuc.lib.siu.edu/phys_pubs

(C) 2003 American Institute of Physics

Published in Journal of Applied Physics, Vol. 93 No. 10 (2003) at doi: 10.1063/1.1555971

\section{Recommended Citation}

Dubenko, I. S., Rao, M. S., Roy, S., Dave, B. C. and Ali, N.. "Magnetic Behavior of Iron-Oxoclusters Prepared in an Organosilica Sol-Gel Matrix." (May 2003).

This Article is brought to you for free and open access by the Department of Physics at OpenSIUC. It has been accepted for inclusion in Publications by an authorized administrator of OpenSIUC. For more information, please contact opensiuc@lib.siu.edu. 


\title{
Magnetic behavior of iron-oxoclusters prepared in an organosilica sol-gel matrix
}

\author{
I. S. Dubenko a) \\ Department of Physics, Southern Illinois University, Carbondale, Illinois 62901 \\ M. S. Rao \\ Department of Chemistry and Biochemistry, Southern Illinois University, Carbondale, Illinois 62901 \\ S. Roy \\ Department of Physics, Southern Illinois University, Carbondale, Illinois 62901 \\ B. C. Dave \\ Department of Chemistry and Biochemistry, Southern Illinois University, Carbondale, Illinois 62901 \\ N. Ali \\ Department of Physics, Southern Illinois University, Carbondale, Illinois 62901
}

(Presented on 14 November 2002)

\begin{abstract}
The crystal structure and magnetization of nanoscale enTMOS- $\mathrm{Fe}_{2} \mathrm{O}_{3}$ sol-gel composites with weight iron concentration $x$, varying from 0.003 to 0.065 , have been studied by the transmission electron microscopy technique and a superconducting quantum interference device magnetometer. The clusters are crystallized in a hexagonal crystal structure. All the samples demonstrate a superparamagnetic behavior with antiferomagnetic cluster-cluster coupling at low temperature. The effective paramagnetic moment, $\mu_{\text {eff }}$, has been found to vary in the range from $5.9(S=5 / 2)$ to 2.5 $\mu_{B}$ per iron ion. The concentration dependence of the $\mu_{\text {eff }}$ shows a minimum for $x \sim 0.01$. At a low iron concentration $x<0.01, \mu_{\text {eff }}$ is practically independent of $x$ and equals about $6 \mu_{B}$ per Fe ion. The concentration interval $0.01<x<0.07$ is characterized by a monotonical increase of $\mu_{\text {eff }}$ from 2.5 to about $3 \mu_{B}$ per Fe ion. Thus, an abrupt variation of $\mu_{\text {eff }}$ (about two times) is observed at $x \approx 0.01$. It has been shown that such behavior can be caused by competition between the uncoupled "surface" and antiferromagnetically coupled "bulk" Fe magnetic moments. (C) 2003 American Institute of Physics. [DOI: 10.1063/1.1555971]
\end{abstract}

\section{INTRODUCTION}

Experimental and theoretical studies of nanoparticle composites show that their magnetic properties are mainly determined by strong intracluster, and relatively weak cluster-cluster exchange interactions. ${ }^{1-6}$ The cluster magnetic ground state can be varied from antiferromagnetic to ferromagnetic depending on the intracluster parameters such as the space configuration of the atomic spins, cluster size, and magnetic ion concentration. Accordingly, the clustercluster exchange interaction can also vary in a wide range to exhibit a very diverse behavior of the nanocluster compounds. ${ }^{7-12}$ Ferritin represents an important class of molecule for magnetic nanostructured composite materials. A single ferritin molecule consists of a protein shell [comprising of 24 subunits with a combined molecular weight of $474000 \mathrm{~g} / \mathrm{mol}$ (Ref. 13)], encapsulating an about 8-nmdiameter-sized spherical core. This core is capable of accumulating $\sim 4000-5000 \mathrm{Fe}$ atoms forming a magnetic cluster. ${ }^{14}$ Numerical simulations have shown that the Fe atoms take up positions on the core surface first. After some limiting concentration, they start to enter into the core to form "bulk" Fe clusters. ${ }^{15}$ Hence, a system that allows a controlled growth of the iron oxocluster with respect to iron

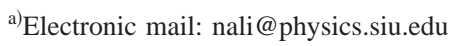

concentration can provide a good opportunity to study the interrelation between the magnetic properties of "surface" and "bulk" nanoclustered systems.

In this article, the magnetic characteristics of an ironoxide nanocluster system prepared in situ by self-assembly of iron oxides within the nanopores of an organically modified silica sol-gel matrix (enTMOS- $\mathrm{Fe}_{2} \mathrm{O}_{3}$ sol-gel composites) are reported. The aim of this work was to study the regularities in the variation of the magnetic properties of iron-oxide nanoclusters as a function of the iron concentration.

\section{EXPERIMENTAL}

The enTMOS- $\mathrm{Fe}_{2} \mathrm{O}_{3}$ sol-gel composites of ironoxonanoclusters within the pores of the sol-gel network (matrix) with different iron concentrations have been prepared from bis[3-(trimethoxysilyl)-propylethylenediamine by the procedure described in detail in Ref. 16. The structural morphology of the clusters studied using the transmission electron microscopic (TEM) technique shows that the clusters having nearly spherical shape with average diameter $(3.8-6.6) \mathrm{nm}$ are isolated from each other by the sol-gel matrix. ${ }^{16}$ The iron concentration in the enTMOS $-\mathrm{Fe}_{2} \mathrm{O}_{3}$ solgel composites has been studied by an atomic emission spectrometer (AES) at a wavelength of $259.94 \mathrm{~nm}$. The samples 


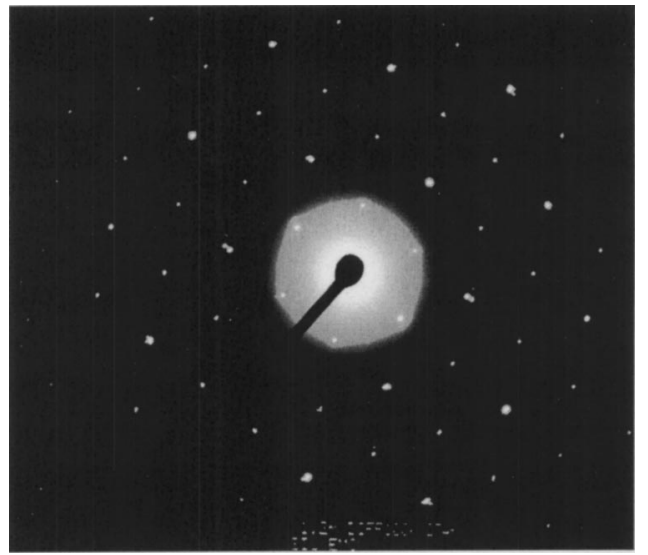

FIG. 1. Typical e-diffraction pattern obtained from the clusters of the enTMOS- $\mathrm{Fe}_{2} \mathrm{O}_{3}$ sol-gel composite (sample N2).

for the AES measurements have been prepared by dissolving a known amount of the enTMOS- $\mathrm{Fe}_{2} \mathrm{O}_{3}$ sol-gel pieces in $50 \mathrm{~mL}$ of $2.5 \% \mathrm{HF}$. The actual iron concentration has been determined from a calibration plot obtained from the AES analysis of a series of standard solutions. Core crystal structures have been observed by the e-diffraction method using TEM. The magnetization in the temperature range 4-300 K of seven selected samples (labeled 1-7) with variation of iron concentration $(x)$ from 0.03 to 0.065 has been measured by a superconducting quantum interference device (SQUID) magnetometer in fields up to $55 \mathrm{kOe}$.

The typical e-diffraction image of the enTMOS- $\mathrm{Fe}_{2} \mathrm{O}_{3}$ sol-gel composites is shown in Fig. 1. As can it be seen the clusters have a hexagonal structure.

In Fig. 2 the magnetization curves of enTMOS- $\mathrm{Fe}_{2} \mathrm{O}_{3}$ sol-gel composites at $5 \mathrm{~K}$ are shown. All of them are characterized by nonlinear-type dependencies of $M(H)$ without spontaneous magnetization. The magnetic moment at $55 \mathrm{kOe}$ depends on the $\mathrm{Fe}$ concentration and increases about ten times from 0.067 to $0.63 \mu_{B}$ per Fe ion for samples N3 and N7 $(x=0.003)$, respectively. Taking into account that the magnetic susceptibility, $\chi$, of these compounds does not show anomalies that could be related to a long distance magnetic ordering in the whole temperature interval $(4.2-300) \mathrm{K}$

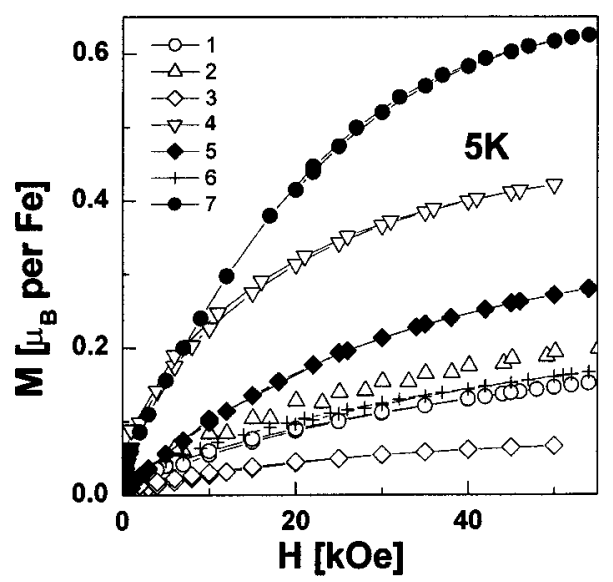

FIG. 2. Magnetization curves of the enTMOS- $\mathrm{Fe}_{2} \mathrm{O}_{3}$ sol-gel composites with different $\mathrm{Fe}$ concentrations at $5 \mathrm{~K}$.

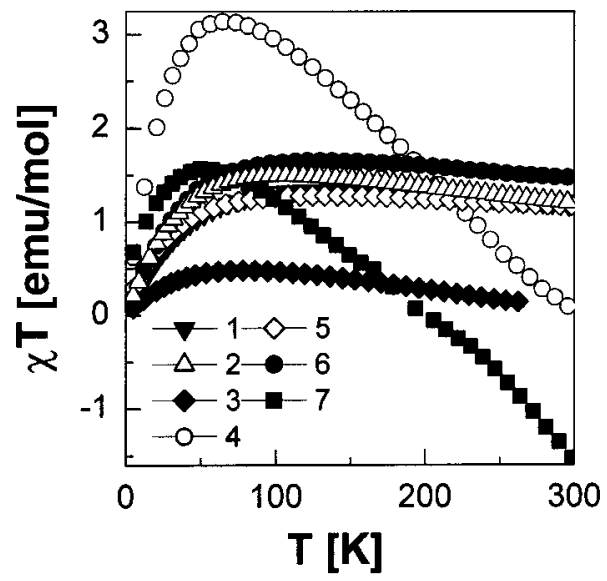

FIG. 3. Temperature dependence of Curie constant $C(T)=\chi T$ for the enTMOS $-\mathrm{Fe}_{2} \mathrm{O}_{3}$ sol-gel composites obtained at $H=10 \mathrm{kOe}$.

(see, also, Ref. 16), it can be concluded that the samples are paramagnets with different magnetic moments (see Fig. 2).

The temperature dependencies of the Curie constant $C(T)=\chi T$ are given in Fig. 3 for 1-7 samples. These dependencies show a broad maximum varying in its peak from $3.12(\mathrm{~N} 4)$ to about $0.5 \mathrm{emu} / \mathrm{mol}(\mathrm{N} 3)$, and approaches zero in low temperature region $T<20 \mathrm{~K}$. Since $C(T) \sim \mu_{\text {eff }}^{2}(T)$, such behavior at low temperature indicates the dominant character of the antiferromagnetic cluster-cluster interaction in this temperature interval. In the high temperature region, where the cluster-cluster interaction is negligible, $\chi(T)$ of enTMOS- $\mathrm{Fe}_{2} \mathrm{O}_{3}$ sol-gel composites is determined by two contributions arising from magnetic susceptibilities of paramagnetic iron clusters, $\chi_{\mathrm{Fe}}$, and of diamagnetic silica and the sol-gel matrix, $\chi_{D} \cdot \chi(T)$ of such systems can be written as

$$
\chi(T)=\chi_{D}+\chi_{\mathrm{Fe}}=\chi_{D}+C_{C} / T,
$$

where $C_{C}$ is the intracluster Curie constant. Thus, $C(T)$ $=\chi T$ will be a linear function of temperature when $\chi_{D}$ is constant.

As it can be seen from Fig. 3, the observed $C(T)$ dependencies at $H=10 \mathrm{kOe}$ follow Eq. (1) in the high temperature region $(T>150 \mathrm{~K})$, and the paramagnetic Curie constant for iron clusters can be evaluated by extrapolation of the linear part of $C(T)$ to zero temperature.

Using the paramagnetic effective magnetic moment of iron clusters obtained from Fig. 3 and Eq. (1), the $\left(\mu_{\text {eff }}-x\right)$ magnetic phase diagram has been constructed (see Fig. 4). Figure 4 shows two different types of behavior of $\mu_{\mathrm{off}}(x)$ in the iron concentration intervals $x<0.01$ and $0.01<x<0.07$, respectively. At the low iron concentration $x<0.01, \mu_{\text {eff }}$ is practically independent of $x$ and equals about $6 \mu_{B}$ per Fe ion. The second concentration interval is characterized by the monotonic increas of $\mu_{\text {eff }}$ from 2.5 to about $3 \mu_{B}$ per Fe ion. Thus, an abrupt variation of $\mu_{\text {eff }}$ (about two times) is observed at $x \approx 0.01$. Taking into account that the value of $\mu_{\text {eff }}=6 \mu_{B}$ is in accordance with the spin number $S=5 / 2$ for "free" $\mathrm{Fe}^{3+}$, the magnetic moments of $\mathrm{Fe}$ ions in clusters are uncoupled in the first concentration interval, while a sharp decreasing of $\mu_{\text {eff }}$ at $x \approx 0.01$ indicates arising of a negative $\mathrm{Fe}-\mathrm{Fe}$ interaction in the next concentration interval. 


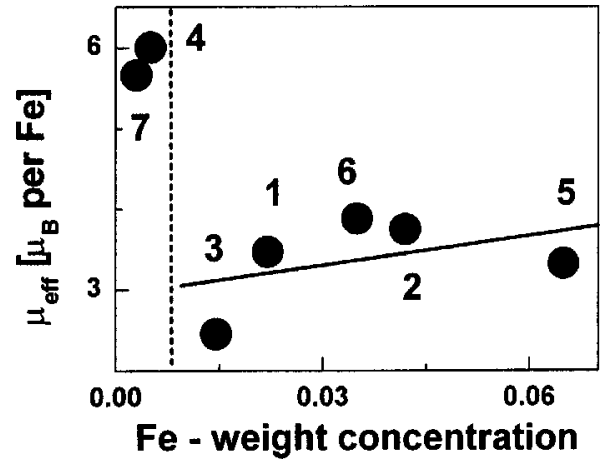

FIG. 4. Concentration dependence of $\mu_{\text {eff }}$ obtained for iron clusters of the enTMOS- $\mathrm{Fe}_{2} \mathrm{O}_{3}$ sol-gel composites. The vertical dash line shows the border between the "surface" and "bulk" clusters.

\section{CONCLUDING REMARKS}

The nanoscale enTMOS- $\mathrm{Fe}_{2} \mathrm{O}_{3}$ sol-gel composites in the iron concentration range $0.003-0.065$ are superparamagnets with the total paramagnetic moment depending on iron concentration and temperature. The magnetic behavior of the composites is determined by negative cluster-cluster coupling and the variable (by $\mathrm{Fe}$ concentration) intracluster interaction of Fe magnetic moments at low and high temperatures, respectively.

The fact that the two intervals can be selected in terms of iron concentration and magnetic properties indicates that the main factor in stabilizing the type of intraclusters magnetic structure is the iron concentration. One can suggest that for iron concentration less than 0.001 , the magnetic moments of Fe ions forming the cluster surface are uncoupled, and as a result the effective magnetic moment does not depend on Fe concentration. The sharp decrease of the magnetic moment around $x=0.01$ can be due to strong negative coupling between iron magnetic moments when the incoming $\mathrm{Fe}$ ions start to enter into the core to form "bulk" Fe clusters. Due to the increased amount of clusters, there is an increasing in the total clusters surface. The resulting uncompensated "surface" Fe ions are responsible for increasing of effective clusters magnetic moment in the second concentration interval.

\section{ACKNOWLEDGMENTS}

This work was supported by the Consortium for Advanced Radiation Source, University of Chicago and grants from Materials Technology Center at SIU, Carbondale (BCD).

${ }^{1}$ R. H. Kodama, J. Magn. Magn. Mater. 200, 359 (1999).

${ }^{2}$ B. A. Grzybowski, H. A. Stone, and G. M. Whitesides, Nature (London) 405, 1033 (1999).

${ }^{3}$ G. A. Prinz, J. Magn. Magn. Mater. 200, 57 (1999).

${ }^{4}$ J. Li, C. Papadopounds, and J. Xu, Nature (London) 402, 253 (1999).

${ }^{5}$ F. J. Himpsel, K. N. Altmann, G. J. Mankey, J. E. Ortega, and D. Y. Petrovykh, J. Magn. Magn. Mater. 200, 456 (1999).

${ }^{6}$ E. H. Lan, B. Dave, J. S. Valentine, and J. I. Zink, Mater. Res. Soc. Symp. Proc. 371, 267 (1995).

${ }^{7}$ B. Martínez, A. Roig, E. Molins, T. González-Carreño, and C. J. Serna, J. Appl. Phys. 83, 3256 (1998).

${ }^{8}$ A. M. Testa, S. Foglia, L. Suber, D. Fiorani, Ll. Casas, A. Roig, E. Molins, J. M. Grenèche, and J. Tejada, J. Appl. Phys. 90, 1534 (2001).

${ }^{9}$ J. J. Lu, H. Y. Deng, and H. L. Huang, J. Magn. Magn. Mater. 209, 37 (2000).

${ }^{10}$ K. W. Edmonds, C. Binns, S. H. Baker, M. J. Maher, S. C. Thornton, O. Tjernberg, and N. B. Brookes, J. Magn. Magn. Mater. 220, 25 (2000).

${ }^{11}$ J. M. L. Billas, A. Chatelain, and W. A. de Heer, Science 265, 1682 (1994).

${ }^{12}$ S. A. Makhlouf, F. T. Parker, F. E. Spada, and A. E. Berkowitz, J. Appl. Phys. 81, 594 (1997).

${ }^{13}$ M. J. Donlin, R. F. Frey, C. Putnam, J. K. Proctor, and J. K. Bashkin, J. Chem. Educ. 75, 437 (1998).

${ }^{14}$ N. D. Chasteen and P. M. Harrison, J. Struct. Biol. 126, 182 (1999).

${ }^{15}$ R. B. Frankel, G. G. Oaoaefthymiou, and G. D. Watt, Hyperfine Interact. 66, 71 (1991).

${ }^{16}$ M. S. Rao, I. S. Dubenko, S. Roy, N. Ali, and B. C. Dave, J. Am. Chem. Soc. 123, 1511 (2001). 